\title{
Movie Recommendation Based on Posters and Still Frames using Machine Learning
}

\author{
S. Muthamil Selvan, Maneesh Pudhota, Sridhar Gunnam, Kaushik Kumar GP
}

\begin{abstract}
Movie recommendation system has become a key part in online movie services to gain and maintain the huge market. While within the preceding studies works Convolution neural network (CNN) concept is employed to spot the various movies with similar posters or stills to recommend the users. Using CNN, similar posters and stills are classified into group and any hard cash within the poster may place it out of the group. But the CNN method isn't fully connected and uses backpropagation technique which could be a touch slow within the poster identification and more over just with posters the films cannot be of comparable one and should disappoint the user. Technologies like Fully Convoluted neural network (FCN) makes use of Convolution neural network concept by connecting all neural networks and adding filters and pooling layer in between each filter layer. Data Augmentation is an algorithm which helps in increasing accuracy for the predicting movies. LASSO regression is employed to get images of high multicollinearity. Soft-max layer is employed to work out the probability of the similarities int poster to create it more appropriate for the user. K-means clustering is employed to classify the films still further to recommend thes implest movietotheuser.
\end{abstract}

Keywords: Fully convoluted neural network, Lasso regression, K means, Soft-max

\section{INTRODUCTION}

The movie recommendation is finished using convolution neural networks. because the convolution neural network isn't fully connected so, it's a touch slow process. The CNN itself cannot reduce the number of parameters which results in make the analysis very complex and increases the time complexity. In general, images of huge sizes cannot to be as an input, it restricts the neuron to think about it. CNN algorithm while implementing might lose the spatial information regarding image which cannot help in accurate prediction. The computational cost of this algorithm is incredibly high. The CNN technique will recommend movies using still posters but with sure error percent, it'll never recommend movie accurately. Fully Convolution neural network (FCN) may be a technique from Deep Learning [3]. FCN technique facilitates in better movie recommendation.

Revised Manuscript Received on April, 252020.

* Correspondence Author

Mr. S. Muthamil Selvan*, Department of Computer Science and Engineering, SRM Institute of Science and Technology, Chennai, India.

Maneesh Pudhota, Department of Computer Science and Engineering, SRM Institute of Science and Technology, Chennai, India.

Sridhar Gunnam, Department of Computer Science and Engineering, SRM Institute of Science and Technology, Chennai, India.

Kaushik Kumar GP, Department of Computer Science and Engineering, SRM Institute of Science and Technology, Chennai, India.

(c) The Authors. Published by Blue Eyes Intelligence Engineering and Sciences Publication (BEIESP). This is an open access article under the CC BY-NC-ND license (http://creativecommons.org/licenses/by-nc-nd/4.0/)
The FCN algorithm has deeper neural layer which stores morecrucial information. There aren't any dense layers in it compared to CNN.The FCN algorithm itself reduces the parameters so, it decreases the time complexity and also the process is a smaller amount complex. Data augmentation is employed which normalizes the identical images of various size, recognition of the other object presents within the image, etc., which allows in higher information of the image that's used within the analysis. FCN first layer which extracts features from the input image and saves its relationship between pixel with the help of constant studying of input image features. Filters are utilized in FCN, that's operated through Kernel operation between Neural networks layers to induce higher characteristic map as output which enables in higher aspect detection. LASSO regression shrinks the information into its central point and helps to develop a straightforward and sparse model image which are easy to interpret. LASSO regression helps avoiding negative valued parameters or less valued parameters. SoftMax function may be a sort of squashing function. This function limits the output of the function into the range 0 to 1 . This helps in allowing the output to be interpreted directly as a probability function output. SoftMax functions are the multi-class sigmoid function, it means they're utilized in determining probability of multiple classes directly. because the output generated by SoftMax layer is interpreted as a probability value, so this layer is often placed eventually stage as final layer in neural networks. it's important to notice that a SoftMax layer must have the identical number of nodes because the output-later. k-means clustering may be a vector quantization method, that's popular for cluster analysis in data processing. k-means clustering main objective is to form $\mathrm{k}$ number of groups by dividing the observation list where each observations are grouped as one based on the Euclidean distance closest mean.

\section{LITERATURE SURVEY}

[1] The movie recommendation system has employed collaborative filtering algorithm. Latent factor model's application within the movie recommendation system improves the system to beat the shortcoming problems in recommending movies. Latent factor model is employed to unravel the matter arises because of sparseness and this method is predicated on non-negative matrix factorization. during this model, the representation of latent factors is completed by using latent factors which describes about the movie.

Published By:

Blue Eyes Intelligence Engineering

\& Sciences Publication

DOI: 10.35940/ijeat.D7255.049420

Journal Website: www.ijeat.org

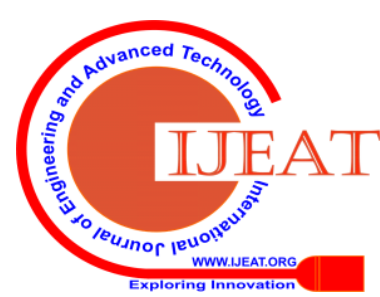


[2] There are different categories of flicks called as genres and are of various languages and cultures present. It creates issue while recommending movies automatically to the users. This paper proposes the $\mathrm{K}$ means algorithm which could be a machine learning algorithm to make the cluster of flicks to separate the genres and similar users to recommend the flicks. It also helps find users with similar taste of flicks. This Machine learning approaches is employed to cluster the flicks of comparable type. The parameters are collected from the dataset are utilized in the network build using Bayesian concept which helps in analysing the user most favoured item. [3] The machine learning field from computer science helps the system to be told and execute automatically supported its selfexperience. In this paper, the movie recommendation system is developed by using algorithms like $\mathrm{K}$ means clustering and $\mathrm{K}$ nearest neighbour algorithm and ContentBased Filtering are explained in details over their usage in this paper. The various clusters and root mean square values are used to find how the algorithm is working and their effectiveness is checked. By reducing the RMSE value to as low as possible will increase the efficiency of the algorithm. The output generated by implementing the proposed work using RMSE has resulted in better performance the using collaborative filtering Technique. Clustering will help in grouping movies based on similar type into one which enhance the viewers the concept.

[4] Automatic recommendation system have become a vital factor for online streaming services platforms like Hotstar and Amazon prime etc. during this paper, we propose collaborative algorithm in predicting movies and also to support movie ratings obtained from different users. As the different content please different users of different perspective of visualisation of movie content. So, the collaborative filtering algorithm moto is to predict movie to a user from unkown users ratings. The deep learning concept enhances the accuracy of algorithm in comparison with standard collaborative filtering algorithm. The proposed method to recommend movies has outperformed the existing systems in terms of both RMSE values and sqaured mean error in the survey research. [5] Information retrieved from movies is growing rapidly because of the large amount of the visual data generated day to day. It not only helps in searching movies in efficient manner by archiving and classification of flicks but it also suggests movies supported the content censorship. The key information from movies are collected and are summarised and added to movies as tags which helps in describing the flicks at their best as compared to other techniques and tagging algorithm is used for better grouping based on English vocabulary. The technique mentioned grasps features from movie frames and uses them to train CNN classifiers. The proposed has undergone a rigorous experimental training process with many various movies. The result obtained by using this proposed system has outperformed the opposite models and also the results of-high-accuracy. [6]Many movie recommendation algorithms are looking affluent visual content of posters and stills which can be parsed to get every minute details which can be used for prediction. The contents of posters are traversed to get the spatial data which is used as input content for filtering algorithm. Recently, no papers are focused on visual contents but they showed more interest in reviews and ratings. But this paper showed a visual utilisation of images and other graphic content for enhancing the movie prediction algorithm. A unique algorithm is used ,unified visual contents matrix factorization (UVMF), where its main aim is to combine all the visual data and other parametrical features into a single framework.

\section{PROPOSED ARCHITECTURE}

\section{A. Input:}

The input module holds movie reviews and well as movie posters.

\section{B. K means:}

$\mathrm{K}$ means algorithm is used to cluster movies based on reviews and genres. It groups the similar reviewed movies. The observations are partitioned into $\mathrm{n}$ observations to reduce within-cluster sum of square.

\section{Data Augmentation:}

Data augmentation is used get images of data from various angles and with varistion to train the system so it can recognise if image is taken from any side with any angle of person.

\section{Fully Convolutional Neural Network:}

this algorithm is used to grasps the image feature and reduces the feature parameters. It obtains the features and constructs image very accurate to ground-truth and reframes it to the original image size

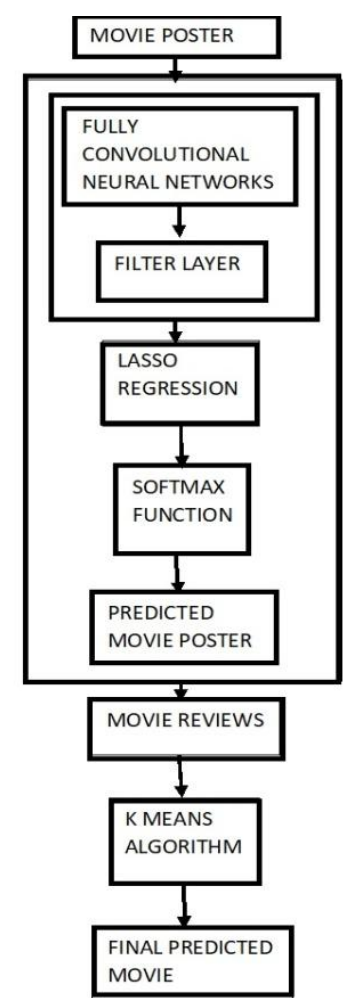

Fig:1 Proposed Architecture of Fully Convolutional neural network method

Published By:

Blue Eyes Intelligence Engineering

\& Sciences Publication

(C) Copyright: All rights reserved. 


\section{E. Lasso Regression and SoftMax:}

Lasso regression reduce the parameters to extract important features which will add more information and to produce the interpretable output movie poster. SoftMax function is used to generate output value as interpretable probability value to decide whether the poster is matching or not.

\section{F. Output:}

The output generated is the movie predicted using a movie poster.

\section{PROPOSED ALGORITHMS}

\section{A. Fully Convolutional Neural Network:}

The Movie Poster with feature map are used to grab the highly crucial details which will be further mapped with backpropagation technique to add the missing details if occurs [18].

$\left(X^{*} Y\right) a b=\sum J 1-1 m=0 \sum J 2-1 n=0 Y m, n . X a+m, b+n+e$

$\mathrm{X}=$ Input image map.

$\mathrm{Y}=$ Filter map bank

$\mathrm{e}=$ biases of $\mathrm{Y}$ filter maps

$\mathrm{m}, \mathrm{n}=$ number of iterations

$\mathrm{k} 1, \mathrm{k} 2$ = kernels

Input image will be transferred to filter map and weights will be assigned to each layer of convolution neural network of values Ym,n.

\section{B. LASSO REGRESSION}

Lasso regression technique holds all the image in vector form which will mapped with feature matrix of training sample to reduce the high parameters used and to do so, it just add the large coefficient of features matrix and avoids the small values or null values or negative values [7]. $\min (0.5|| \mathrm{L}-\mathrm{A} \alpha\|22+\lambda\| \alpha \| 1)$

$L$ is vector holding $M$ labels of training samples and feature map matrix of $\mathrm{M}$ training samples is represented with symbol $\mathrm{A}$ with $\mathrm{N}$ features. $\lambda$ Is the coefficient used with a value of 0.1 to reduce error. $\alpha$ It is the spare coefficient and used to select features with large coefficients

\section{K MEANS CLUSTERING}

$\mathrm{K}(\mathrm{s})(\mathrm{i})=\{\mathrm{Yt}:\|\mathrm{Yt}-\mathrm{l}(\mathrm{s})(\mathrm{i})\| 2<=\|\mathrm{Yt}-\mathrm{l}(\mathrm{s})(\mathrm{j})\| 2$ for all $\mathrm{j}$, $1<=\mathrm{j}<=\mathrm{k}\}$

$1(\mathrm{~s}+1) \mathrm{I}=1 /(\mathrm{K}(\mathrm{s}) \mathrm{i})\left(\sum(\mathrm{Yj})\right.$, limit $\mathrm{Yj}$ belongs to $\left.\mathrm{K}(\mathrm{s}) \mathrm{i}\right)$

$\mathrm{K}$ is sets of partition

$\mathrm{Y}$ is set of observation which is to be clustered

$\mathrm{l}$ is the given set of K-means value i.e. the initial centroids.

\section{SOFTMAX}

$\left(\partial / \partial \mathrm{w}_{\mathrm{i}}\right) \boldsymbol{\sigma}(\mathrm{w}, \mathrm{k})=\boldsymbol{\sigma}(\mathrm{w}, \mathrm{k})\left(\delta_{\mathrm{ki}}-\boldsymbol{\sigma}(\mathrm{w}, \mathrm{i})\right)$

$\mathrm{W}$ is image vector

$\mathrm{k}$ is the real specific index value

$\delta$ Kronecker delta function

SoftMax is used to give interpretable value to declare whether the movie posters are similar to the input movie poster or not.
V.

EXPERIMENTAL RESULTS

Table1: Result of different layers of FCN

\begin{tabular}{|l|l|l|l|l|}
\hline & $\begin{array}{l}\text { Pixel } \\
\text { accuracy }\end{array}$ & $\begin{array}{l}\text { Mean } \\
\text { accuracy }\end{array}$ & Mean IU & F.W. IU \\
\hline $\begin{array}{l}\text { FCN-32s } \\
\text { fixed }\end{array}$ & 82.9 & 58.4 & 46.74 & 71.1 \\
\hline FCN-32s & 90.12 & 76.28 & 58.64 & 82.44 \\
\hline FCN-16s & 91.15 & 77.74 & 61.14 & 82 \\
\hline FCN-8s & 92.73 & 76.09 & 63.77 & 84.12 \\
\hline
\end{tabular}

IU (Intersection over Union) score is calculated as

(Truth positive)/ (Truth positive + False positive + False negative). Mean IU is the average value of the all IU scores.

- pixel accuracy: $\sum_{\mathrm{i}} \mathrm{n}_{\mathrm{ii}} / \sum_{\mathrm{i}} \mathrm{t}_{\mathrm{i}}$

- mean accuracy: $\left(1 / \mathrm{n}_{\mathrm{cl}}\right) \sum \mathrm{n}_{\mathrm{ii}} / \mathrm{t}_{\mathrm{i}}$

- mean IU: $\left(1 / \mathrm{n}_{\mathrm{cl}}\right) \sum_{\mathrm{i}} \mathrm{n}_{\mathrm{ii}} /\left(\mathrm{t}_{\mathrm{i}}+\sum_{\mathrm{j}} \mathrm{n}_{\mathrm{ji}}-\mathrm{n}_{\mathrm{ii}}\right)$

- frequency weighted IU:

$\left(\sum_{\mathrm{k}} \mathrm{t}_{\mathrm{k}}\right)^{-1} \sum_{\mathrm{i}} \mathrm{t}_{\mathrm{i}} \mathrm{n}_{\mathrm{ii}} / \quad\left(\mathrm{t}_{\mathrm{i}}+\sum_{\mathrm{j}} \mathrm{n}_{\mathrm{ji}}-\mathrm{n}_{\mathrm{ii}}\right)$ $\mathrm{t}_{\mathrm{i}}=\sum_{\mathrm{j}} \mathrm{n}_{\mathrm{ij}}$.

Table2: Results Comparison between CNN and FCN

\begin{tabular}{|l|l|l|}
\hline & Mean IU & $\begin{array}{c}\text { Inferenc } \\
\text { e Time }\end{array}$ \\
\hline CNN & 47.9 & $50 \mathrm{~s}$ \\
\hline FCN-8s & 62.7 & $175 \mathrm{~ms}$ \\
\hline
\end{tabular}

Inference time is the time used to predict the movie by classifying posters using machine learning algorithms. The obtained pixel accuracies, Mean IU and F.W. IU values are increasing as unsampled stride value is decreasing. The lower the value the more is the accuracy and IU values as the image undergoes more unsampling process to get larger image.

\section{CONCLUSION}

The proposed method works in a unique way to increase Pixel and Mean accuracy, Mean and Frequency weighted IU compared to the convolution layer. The Fully convoluted neural network has captured better detailing of the movie posters and frames etc. and those details are used to aid in predicting movie. In Table2, the observed Mean IU value of Fully convoluted neural network is higher than Convolution neural network and the Interface time of FCN is very low compared to other methods. The Pixel accuracy from Table1 states that FCN is best algorithm in comparing or classifying images by factoring pixels.LASSO regression for simple and interpretable imagewith an accuracy of $98.10 \%$. SoftMax algorithm helps in deciding whether the given movie poster is similar to the original given movie poster at a faster rate. The $\mathrm{K}$ means algorithm is an unsupervised algorithm which is used to cluster similar movies by grouping them based on similar ratings.

\section{REFERENCES}

1. Liu, W., Wang, B., \& Wang, D. (2018). Improved Latent Factor Model in Movie Recommendation System. 2018 International Conference on Intelligent Autonomous Systems (ICoIAS). doi:10.1109/icoias.2018.8494074

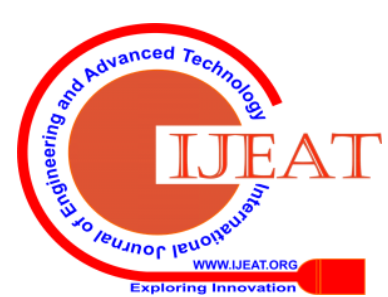




\section{Movie Recommendation Based on Posters and Still Frames using Machine Learning}

2. Ahuja, R., Solanki, A., \& Nayyar, A. (2019). Movie Recommender System Using K-Means Clustering AND K-Nearest Neighbor. 2019 9th International Conference on Cloud Computing, Data Science\&Engineering.(Confluence). doi:10.1109/confluence.2019.87 76969

3. Lund, J., \& Ng, Y.-K. (2018). Movie Recommendations Using the Deep Learning Approach. 2018 IEEE International Conference on Information Reuse and Integration (IRI). doi:10.1109/iri.2018.00015

4. Khan, U. A., Ejaz, N., Martinez-del-Amor, M. A., \&Sparenberg, H. (2017). Movies tags extraction using deep learning. 2017 14th IEEE International Conference on Advanced Video and Signal Based Surveillance (AVSS). doi:10.1109/avss.2017.8078459

5. Exploiting Visual Contents in Posters and Still Frames for Movie Recommendation

6. Published in: IEEE Access (Volume: 6 ), Page(s): 68874 - 68881, Date of Publication: 09 November 2018, Electronic ISSN: 21693536DOI: 10.1109/ACCESS.2018.2879971

7. Backpropagation In Convolutional Neural Networks, Jefkine, 5 September 2016

8. Improving Recommender Systems by IncorporatingSocial Contextual Information,Author:Tom Chao Zhou. Authors Info \& Affiliations, Publication:ACM Transactions on Information Systems (TOIS)April 2011 ArticleNo.:9 https://doi.org/10.1145/1961209.1961212

9. The role of user mood in movie recommendationsExpert Systems with ApplicationsVolume 37, Issue 8, August 2010, Pages 60866092, https://doi.org/10.1016/j.eswa.2010.02.117

10. Wu, C.-S. M., Garg, D., \&Bhandary, U. (2018). Movie Recommendation System Using Collaborative Filtering. 2018 IEEE 9th International Conference on Software Engineering and Service Science (ICSESS). doi:10.1109/icsess.2018.8663822

11. Kumar Manoj, D.K. Yadav, Singh Ankur, Kr Vijay, "A Movie Recommender System: MOVREC", 2015 International Journal of Computer Applications, vol. 124, pp. 7-11.

12. H. W. Chen, Y. L. Wu, M. K. Hor, C. Y. Tang, "Fully content-based movie recommender system with feature extraction using neural network", 2017 International Conference on Machine Learning and Cybernetics (ICMLC), pp. 504-509, 2017.

13. S. K. Ko et al., "A smart movie recommendation system," Human Interface and the Management of Information. Interacting with Information, pp. 558-566, 2011.

14. X. Wang, F. Luo, C. Sang, J. Zeng, and S. Hirokawa, "Personalized Movie Recommendation System Based on Support Vector Machine and Improved Particle Swarm Optimization," IEICE TRANSACTIONS on Information and Systems, vol. 100, no. 2, pp. 285-293, 2017.

15. V. Chandrasekaran, S. Sanghavi, P. A. Parrilo, and A. S. Willsky, "Rank-sparsity incoherence for matrix decomposition," SIAM Journal on Optimization, vol. 21, no. 2, pp. 572-596, 2011.

16. H. Drachsler, T. Bogers, R. Vuorikari, K. Verbert, E. Duval, N. Manouselis, G. Beham, S. Lindstaedt, H. Stern, M. Friedrich, et al. Issues and considerations regarding sharable data sets for recommender systems in technology enhanced learning. Procedia Computer Science, 1(2):2849-2858, 2010.

17. AlvaroTejeda-Lorente, A quality based recommender system to dissem- ' inate information in a university digital library,Information Sciences Volume 261, 10 March 2014, Pages 52-69

18. Trang Tran, T.N., Atas, M., Felfernig, A. et al. J Intell Inf Syst (2018) 50: 501. https://doi.org/10.1007/s10844-017-0469-0

19. Xin Luo, Mengchu Zhou, Yunni Xia, and QingshengZhu,An Efficient Non-Negative Matrix-Factorization-Based Approach to Collaborative Filtering for Recommender Systems,IEEE Transactions on Industrial Informatics ( Volume: 10 , Issue: 2 , May 2014 )

20. J. McAuley, C. Targett, Q. Shi, A. van den Hengel, "Image-based recommendations on styles and substitutes", Proc. 38th Int. ACM SIGIR Conf. Res. Develop. Inf. Retr., pp. 43-52, 2015.

21. Show Context Access at ACM Google Scholar

22. 18. R. He, J. McAuley, "Ups and downs: Modeling the visual evolution of fashion trends with one-class collaborative filtering", Proc. 25th Int. Conf. World Wide Web, pp. 507-517, 2016.

\section{AUTHORS PROFILE}

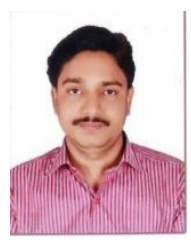

S. MuthamilanSelvan, Institute: SRM Institute of Science and Technolog Chennai-89 Assistant Professor, CSE department and has degree in Master of Engineering

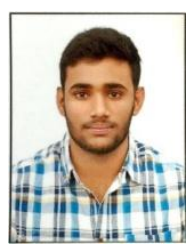

Maneesh Pudhota, Institute: SRM Institute of Science and Technology, Chennai-89. Pursuing B.Tech. in SRMIST

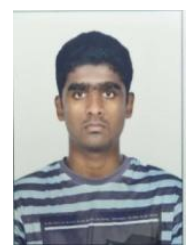

Sridhar Gunnam,Institute: SRM Institute of Science and Technology, Chennai-89. Pursuing B.Tech. in SRMIST

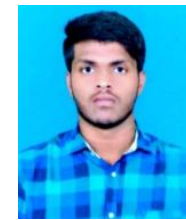

Kaushik Kumar GP, Institute: SRM Institute of Science and Technology, Chennai-89.Pursuing B.Tech. in SRMIST

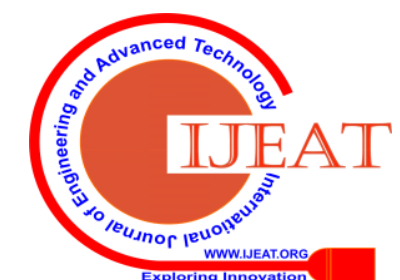

